\title{
Musculoskeletal and nervous systems of the attachment organ in three species of Diplectanum (Monogenea: Dactylogyroidea)
}

\author{
${ }^{1}$ Zoological Institute of the Russian Academy of Sciences, Saint Petersburg, Russia; \\ ${ }^{2}$ A.O. Kovalevsky Institute of Marine Biological Research, Sevastopol, Crimea; \\ ${ }^{3}$ Murmansk Marine Biological Institute, Murmansk, Russia
}

Anatoly A. Petrov ${ }^{1}$, Evgenija V. Dmitrieva ${ }^{2}$, Maryana P. Popyuk ${ }^{3}$, Pavel I. Gerasev ${ }^{1}$ and Sergey A. Petrov

\begin{abstract}
The taxonomy of Diplectanum Diesing, 1858, a genus of monopisthocotylean monogeneans, remains unsettled and needs to be revised based on new morphological criteria. Recent studies in monopisthocotyleans have shown that the muscle arrangement in the posterior attachment organ (haptor) differs between congeneric species and can be used as an additional criterion in genus-level taxonomy. To explore the possibility of using the haptoral musculature and nervous system in the taxonomy of Diplectanum, we conducted a detailed confocal-microscopy study of three species of Diplectanum (D. aculeatum Parona et Perugia, 1889, D. sciaenae van Beneden et Hesse, 1863 and $D$. similis Bychowsky, 1957) with phalloidin staining for muscle and indirect immunostaining for 5HT and FMRFamide. A further goal was to clarify the functional mechanics of the haptor and the role of its essential components (squamodiscs and anchors) in attachment to the host. The system of connecting bars and gaffing anchors was found to have a complex musculature consisting of 23 muscles in D. aculeatum and D. sciaenae, and 21 muscles in D. similis. The squamodiscs were shown to be operated by several groups of muscles attached primarily to the area termed the squamodisc fulcrum. Most of the haptoral musculature is identical in D. aculeatum and D. sciaenae and these species differ only in the presence of a muscle sheath around the tissue strand between the squamodiscs in D. sciaenae and in the different patterns of superficial squamodisc muscles. Diplectanum similis shows more significant differences from the other two species: besides lacking two of the haptoral muscles, it also differs in the shapes and arrangement of several other muscles. The nervous system of all three species conforms to the general pattern typical for the Dactylogyroidea and shows little variation between species.
\end{abstract}

Keywords: Monopisthocotylea, musculature, haptor, phalloidin, 5HT, FMRFamide, squamodisc, anchor

Diplectanum Diesing, 1858 is a genus of monopisthocotylean monogeneans comprising about 70 nominal species (Gibson and Bray 2010), only 14 of which, including the type species $D$. aequans (Wagener, 1857), constitute a strongly supported monophyletic group. The generic placement of the remaining species needs further clarification, which may warrant reassessment of the known morphological characters or identification of new morphological criteria (Domingues and Boeger 2008, Justine and Henry 2010).

The systematics of Diplectanidae relies mostly on characters of the posterior attachment organ (haptor) and distal regions of the reproductive system. Recent studies of the haptoral musculature in two monopisthocotylean genera, Ligophorus Euzet et Suriano, 1977 and Dactylogyrus Diesing, 1850 (see Petrov et al. 2015, 2016), have shown that the arrangement of the haptoral muscles differs between congeneric species and can be used as an additional cri- terion in genus-level taxonomy. The study of the haptoral musculature may also reinforce our knowledge of the morphology of haptoral sclerites, because a significant number of landmarks on sclerites serve as attachment points for muscles and some of the taxonomically-relevant landmarks can be overlooked or misinterpreted without proper understanding of the associated musculature.

The posterior attachment organ of Diplectanum consists of two major components: a pair of attachment discs (squamodiscs) and a set of anchors and connecting bars comprising the gaffing apparatus of the haptor. The available knowledge of the haptoral musculature in Diplectanum spp. is currently limited to the information on some major anchoral muscles in $D$. aequans, $D$. aculeatum Parona et Perugia, 1889 and D. sciaenae van Beneden et Hesse, 1863 (Paling 1966, Oliver 1987) and the general morphology (Paling 1966) and ultrastructure (Shaw 1981) of squamodisc muscles in $D$. aequans. The purpose of the present

Address for correspondence: A.A. Petrov, Zoological Institute of the Russian Academy of Sciences, Universitetskaya Emb. 1, Saint Petersburg, 199034, Russia. Phone: +7(812)3280612; Fax:+7(812)3282941; E-mail: anatoly.petrov@zin.ru 
study was to provide a detailed description of the muscle and nervous systems of the haptor in species of Diplectanum parasitising the brown meagre, Sciaena umbra Linnaeus, in the Black Sea, with an emphasis on species-specific differences in muscle arrangement.

\section{MATERIALS AND METHODS}

Specimens of Diplectanum were collected from Sciaena um-

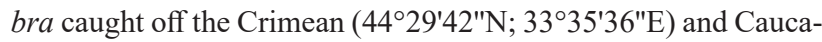

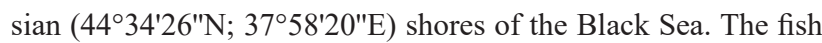
were transported live to the laboratory and examined for monogeneans immediately after killing. The worms collected from the excised gills were fixed immediately with $4 \%$ (w/v) paraformaldehyde in $0.01 \mathrm{M}$ phosphate-buffered saline (PBS) at ambient temperature and after $6 \mathrm{~h}$ of fixation were transferred to $0.01 \mathrm{M}$ PBS containing $0.05 \%(\mathrm{w} / \mathrm{v})$ sodium azide for storage at $4{ }^{\circ} \mathrm{C}$.

All subsequent steps were performed at room temperature on an orbital shaker. The worms were permeabilised for $2-4 \mathrm{~h}$ with $0.25 \%$ Triton X-100 in 0.01M PBS (Tr-PBS) and blocked with Image-iT FX Signal Enhancer (Molecular Probes, Eugene, Oregon, USA) for $1 \mathrm{~h}$. Blocked animals were rinsed $4 \times 15 \mathrm{~min}$ in Tr-PBS, incubated for $10-12 \mathrm{~h}$ in FMRFamide $(1: 600)$ (Abcam, Cambridge, Massachusetts, USA) or 5HT (1:600) (Sigma-Aldrich, St. Louis, Missouri, USA) antibodies, rinsed again $4 \times 15 \mathrm{~min}$ in Tr-PBs and incubated for $5 \mathrm{~h}$ in Alexa Fluor 488-conjugated secondary antibodies $(1: 300)$ (Life Technologies, Carlsbad, California, USA). After incubation, the monogeneans were rinsed in Tr-PBS for 15 min and stained 2-3 h with Alexa Fluor 543-conjugated phalloidin $(1: 200)$ (Life Technologies), then rinsed once in $0.01 \mathrm{M}$ PBS and mounted on slides with Vectashield (Vector Laboratories Inc., Burlingame, California, USA). Staining specificity was checked by omitting primary antibodies.

Confocal images were collected on a Leica TCS SP5 confocal laser scanning microscope at the 'Taxon' Research Resource Centre (Zoological Institute of the Russian Academy of Sciences, Saint Petersburg, Russia). Sclerites were visualised in the reflection mode of the confocal microscope as described in Petrov et al. (2016): the worms were irradiated with a $\mathrm{He}-\mathrm{Ne}$ $(633 \mathrm{~nm})$ laser and the images were acquired at a detection range of 625-645 nm. All confocal images of phalloidin-stained whole mounts were presented as maximum intensity projections of the Z-stacks. Three-dimensional schemes were made using Pixologic ZBrush 4R6. All measurements are in micrometres unless otherwise indicated.

Some of the individuals of Diplectanum spp. collected from the same fish specimens as those used for confocal microscopy were mounted in glycerol-gelatin and deposited as voucher specimens in the collection of parasites of marine fish at the A.O. Kovalevsky Institute of Marine Biological Research (http://marineparasites.org). Species identification was based on published descriptions of species of Diplectanum occurring in the Mediterranean region (Oliver 1968, 1980, 1987, Oliver and Paperna 1984).

Unless explicitly noted, the terminology follows Domingues and Boeger (2008) and Galli et al. (2009); in particular, the anchoral root forming the extension of the blade is referred to as outer root and the root facing the inside of the blade curvature as inner root.

\section{Abbreviations:}

avd4 - anterior branch of $v d 4$-muscle

bs - body scales

$\mathrm{da}$ - dorsal anchor

dad1-3 - muscles connecting dorsal anchor and dorsal bar

dao - muscle connecting dorsal anchor to anchoral opening

das - deep arcuate squamodisc muscles

daw - muscle connecting dorsal anchor to body wall

$\mathrm{db}$ - dorsal bar

$\mathrm{dvc}$ - dorsoventral cylinder

dvs - dorsoventral squamodisc muscles

dw1-3 - muscles connecting dorsal bar to body wall

$\mathrm{fc}$ - squamodisc fulcrum

$\mathrm{idb}$ - muscle connecting opposite dorsal bars

if - interconnecting fulcral muscles

iv - inner root of ventral anchor

ivd1-2 - muscles connecting ventral and dorsal anchors

lbw - longitudinal muscle band in haptor wall

If - longitudinal fulcral muscles

lts - lateral portions of $t s$-muscles

$\mathrm{lvb}$ - lateral tip of ventral bar

lvw - longitudinal branch of $v w$-muscle

$\mathrm{mb}$ - blade of marginal hook

mbs - sickle base of marginal hook

$\mathrm{mc}$ - muscular coat of dorsoventral cylinder

mex - extrinsic muscles of marginal hooks

$\mathrm{mg}-$ muscular wall of anchoral gland

$\mathrm{mh}$ - marginal hook

$\mathrm{mp}$ - papilla of marginal hook

mpp - medio-posterior projection of dorsal bar

$\mathrm{mpr}$ - protractor muscle of marginal hook

$\mathrm{ms}$ - shaft of marginal hook

msa - meshwork-like pattern of sas-muscles

obw - oblique muscle band in haptor wall

od - outer root of dorsal anchor

oda - opening of dorsal anchor

ov - outer root of ventral anchor

ova - opening of ventral anchor

ovw - oblique branch of $v w$-muscle

pvd4 - posterior branch of $v d 4$-muscle

rab - anchoral neurite bundle, RF-IR

rah - anterior segment of haptoral loop, RF-IR

rf - radiating fulcral muscles

$\mathrm{rlb}$ - neurites projecting over lateral portions of bars, RF-IR

rll - lateral longitudinal neurite bundle, RF-IR

rmg - medial ganglion, RF-IR

rnd - neurite bundle of dorsal anchor, RF-IR

$\mathrm{rnr}$ - lateral neurites of squamodisc rows, RF-IR

rpc - peduncular commissure, RF-IR

$\mathrm{rph}$ - posterior segment of haptoral loop, RF-IR

rsb - side branches of squamodisc arborisation, RF-IR

rsc - neuron somata at confluence of neurite bundles, RF-IR

rsp - neuron somata in posterior body proper, RF-IR

rtn - tripolar neuron, RF-IR

rvl - ventral longitudinal neurite bundle, RF-IR

sah - anterior segment of haptoral loop, 5HT-IR

sam - anterior neurite of medial ganglion, 5HT-IR

sas - superficial arcuate squamodisc muscles 


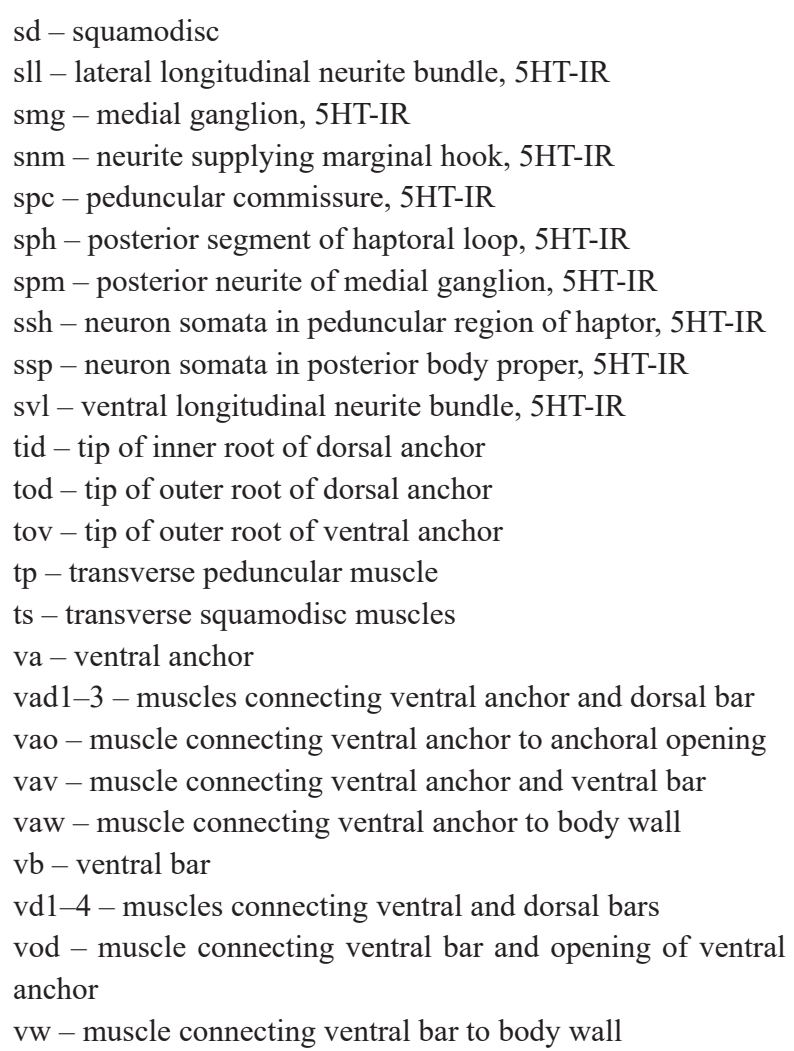

\section{RESULTS}

Three species were identified from the specimens of $\mathrm{Di}$ plectanum collected from Sciaena umbra: D. aculeatum, D. similis Bychowsky, 1957 and D. sciaenae. The latter species is reported from the Black Sea for the first time.

Morphology of haptoral sclerites. The sclerites of the haptor in all species comprise ventral and dorsal squamodiscs ( $s d$, Fig. 1C,F), one unpaired ( $v b$, Figs. 1A-C,E,H, $2 \mathrm{~B}, \mathrm{D})$ and two paired ( $d b$, Figs. $1 \mathrm{~A}-\mathrm{C}, \mathrm{G}, 2 \mathrm{~B}-\mathrm{D}, 3 \mathrm{C}, \mathrm{E}$, $4 \mathrm{~A}, \mathrm{~B})$ transverse bars, one ventral and one dorsal pairs of anchors (Fig. 1A-C) and seven pairs of identical marginal hooks (Fig. 1D; $m h$, Figs. 1F, 3E).

The unpaired medial bar lies ventrally; it tapers to a point at both lateral ends and is attenuated in the middle, with an especially conspicuous anterior notch, which is U-shaped in D. aculeatum (Fig. 1B) (http://marineparasites.org/taxa/?taxon=41) and $\mathrm{V}$-shaped in D. sciaenae (Fig. 1A) (http://marineparasites.org/taxa/?taxon=481) and D. similis (Fig. 1C) (http://marineparasites.org/taxa/?taxon=42). The paired bars are dorsal in position; they are club-shaped, with an enlarged medial portion and a long lateral shaft. In most specimens, the medial ends of the opposite bars butt together or overlap in the middle of the haptor and the bars are oriented at an obtuse angle to each other to form a wide V-shaped configuration.

The anchors lie at the lateral tips of the dorsal bars, with two anchors at each tip comprising one of the ventral and one of the dorsal pair (Fig. 1A-C). The dorsal anchor appears to be directly connected to the lateral end of the dorsal bar with a short ligament, while the ventral anchor has no ligamentous connection to the bar and is attached to it only with muscles. The anchors are sickle-shaped, with a long outer root (ov, od, Fig. $1 \mathrm{~A}-\mathrm{C})$ and a thin curved blade. The inner root of the dorsal anchor is short and appears as a wide trapezoid projection on the inner side of the anchor. One of the corners of this projection (tid, Fig. 1A-C) is an attachment point for one of the anchoral muscles $(i d v 1)$ and is tentatively interpreted here as the distal tip of the root. The inner root of the ventral anchor ( $i v$, Fig. $1 \mathrm{~A}-\mathrm{C}$ ) is short and cylindrical in shape and is oriented at an acute angle with respect to the outer root.

Marginal hooks ( $m h$, Fig. $1 \mathrm{~F}$ ) are unhinged and are subdivided into two regions: a straight proximal shaft ( $m s$, Fig. 1D) with a bulb-shaped extension at its proximal end and a distal sickle composed of a slim arcuate base ( $m b s$, Fig. 1D) and slightly curved blade ( $m b$, Fig. 1D). The hooks open at the tips of small protrusions of the body wall (hook's papillae) ( $m p$, Fig. 1F). Two pairs of marginal hooks are positioned close to the haptor midline behind the connecting bars, while the other five sit along its lateral margins.

The squamodiscs are covered by concentric arch-shaped rows of interlocked rodlets (ossicles) with imbricated outer scale-like extensions (Fig. 1F). The anterior arches are narrow and $\mathrm{V}$-shaped, but posteriorly the rows of ossicles are gradually flattening to almost straight lines. The circular area located about one-third the length of the squamodisc from its anterior margin at the tips of the anterior-most arches is the place of attachment of most squamodisc muscles and is designated here as squamodisc fulcrum $(f c$, Figs. 1F, 2A, 5C). In fixed specimens the squamodiscs are often conically dimpled due to muscle contraction with the fulcra located at the apices of these depressions.

Anchoral musculature. The anchoral musculature comprises 13 major muscles that can be divided into five categories according to their insertion on the ventral or dorsal bars, the body wall, the other ipsilateral anchor or an anchoral opening.

The six muscles inserting on the ipsilateral dorsal bar is the most numerous and prominent group of anchoral muscles. Three of those ( $d a d 1-3)$ have their origin on the dorsal anchor, the other three (vad1-3) originate from the ventral anchor. Dad1 (Figs. 2C,D, 3A-C,E, 4A,B, 5A) is the largest of the anchoral muscles. Extending posteriorly from the tip of the outer root of the dorsal anchor, it reaches the posterior margin of the dorsal bar, wraps around the ventral and anterior aspects of the bar and inserts on the dorsal side of the bar a short distance from its lateral end. Dad2 (Figs. 2D, 3C) is a thick spindle-shaped muscle running from the tip of the outer root of the dorsal anchor to the dorsal bar and inserting on the ventral side of the bar about the same distance from its lateral end as dad1. Dad3 (Figs. 2C, 3B) has a roughly triangular shape, with a fleshy attachment to the outer root on the outer side of the dorsal anchor and a narrow insertion point on the lateral tip of the dorsal bar.

Muscles vad1 and vad2 take their origin from the tip of the outer root of the ventral anchor and run posteriorly to insert on the dorsal side of the dorsal bar. Vad1 (Figs. 2C,D, $3 \mathrm{~B}, \mathrm{C})$ is a wide spindle-shaped muscle that inserts on the bar very close to its lateral tip, just lateral to the insertion of dad1. The attachment spot of both $d a d 1$ and vadl is marked 

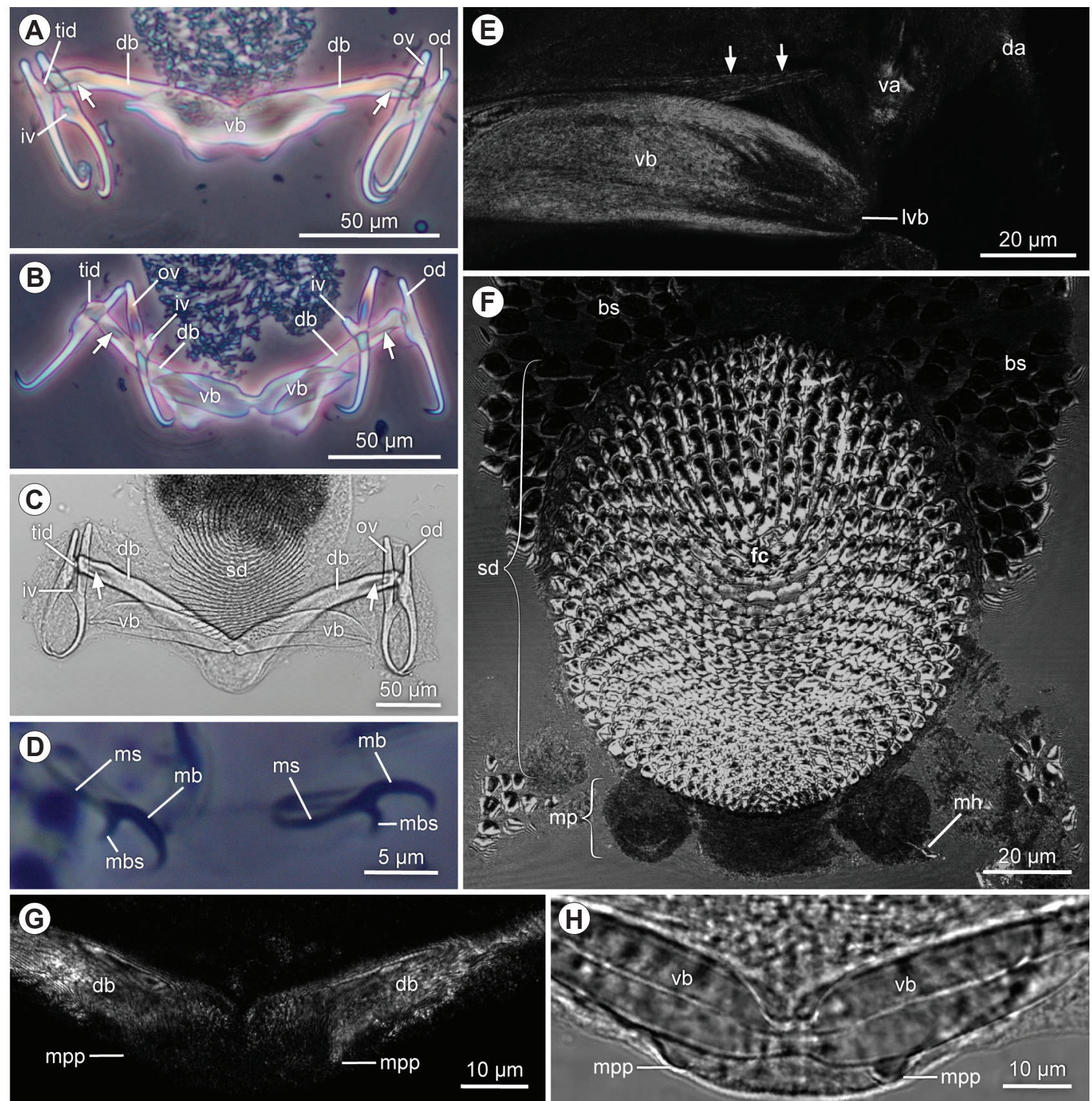

Fig. 1. Morphology of haptoral sclerites of species of Diplectanum Diesing, 1858 from Sciaena umbra Linnaeus. A - phase contrast image of anchors and connecting bars of $D$. sciaenae van Beneden et Hesse, 1863; B - phase contrast image of anchors and connecting bars of $D$. aculeatum Parona et Perugia, 1889; $\mathbf{C}$ - bright-field image of anchors, connecting bars and squamodisc (sd) of $D$. similis Bychowsky, 1957; D - phase contrast images of marginal hooks of D. sciaenae; $\mathbf{E}$ - haptoral sclerites and the tendinous projection of the vad3-muscle (arrows) of $D$. similis in reflected light; $\mathbf{F}$ - posterior body proper and the ventral squamodisc (sd) of $D$. sciaenae in reflected light showing body scales (bs) and squamodisc ossicles; $\mathbf{G}$ - reflected-light image of the central portion of the haptor of D. aculeatum showing the medio-posterior projections (mpp) on the dorsal bars; $\mathbf{H}$ - bright-field image of the central portion of the haptor of D. aculeatum (the same individual as in $\mathrm{G}$ ). Arrows in $\mathrm{A}-\mathrm{C}$ show grooves at the lateral ends of the dorsal bars. For $\mathrm{Abbrevi-}$ ations see pages 2 and 3 .

by a prominent groove on the dorsal side of the bar (arrows, Fig. 1A-C). Vad2 (Figs. 2C, 3A,E, 4A, 5A) is thinner than vad1; it attaches to the dorsal bar farther away from its lateral tip and is partly overlaid by dad1. The insertion of vad3 (Figs. 2C,D, 4A, 5A) is on the oblique ridge that runs ventrally along the medio-posterior extension of the dorsal bar; the fibres of this muscle extend laterally converging on a long and narrow tendinous projection, which lacks actin and can be seen only in reflected light (arrows, Figs. 1E, $4 \mathrm{~A}$ ). The projection reaches the mid-section of the ventral anchor and, although its exact point of attachment is unclear, it most likely attaches to the anchor at the transition between the anchoral blade and the outer root.

The ventral bar is connected to the anchors with one muscle (vav, Figs. 2C,D, 3A,D,E, 5C, 6C). This muscle originates on the outer side of the ventral anchor at about 

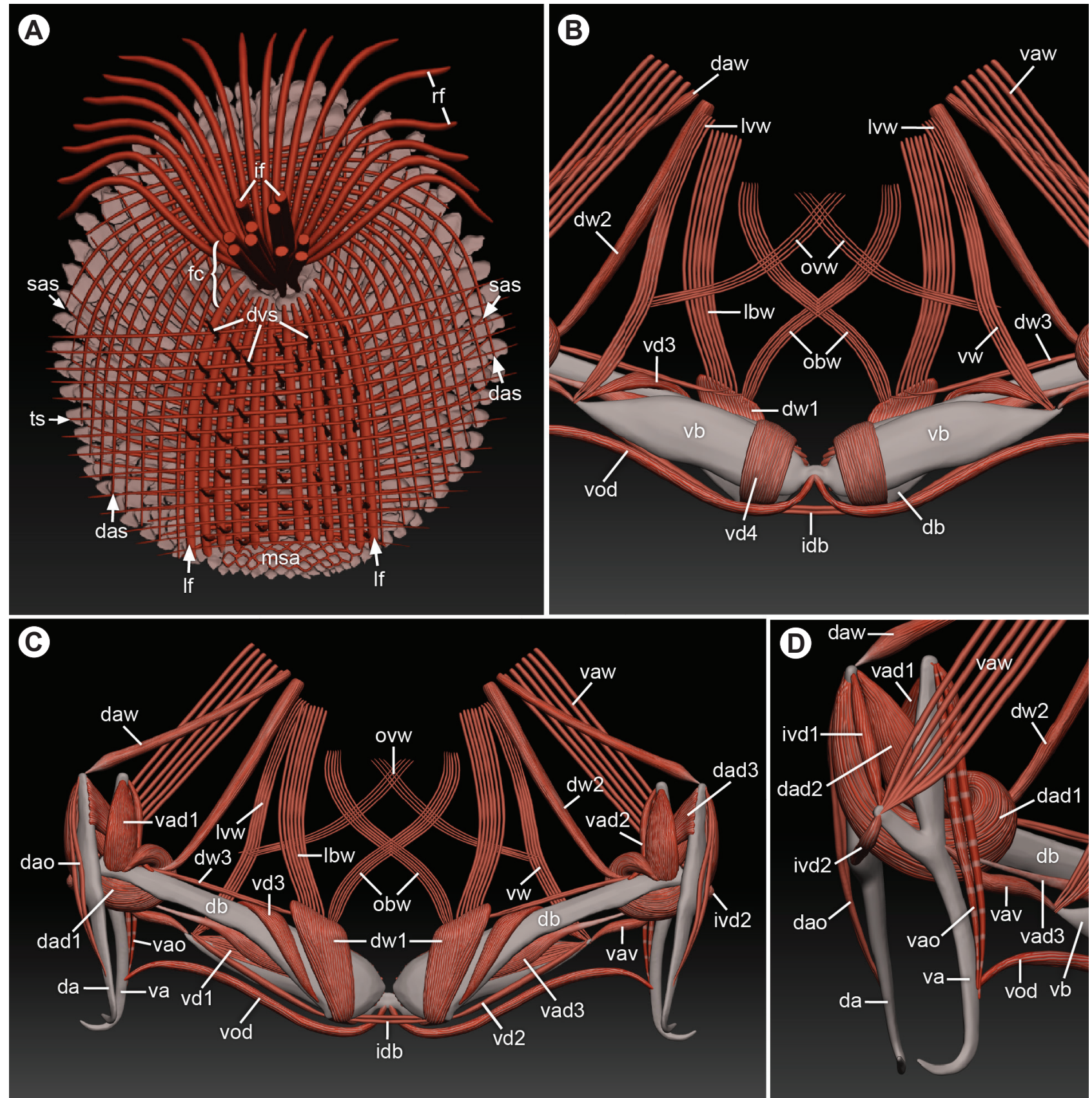

Fig. 2. Schematic representation of haptoral sclerites and musculature of species of Diplectanum Diesing, 1858 from Sciaena umbra Linnaeus. A - squamodisc musculature of D. sciaenae van Beneden et Hesse, 1863 viewed from the interior of the haptor; $\mathbf{B}$ - musculature of the connecting bars of $D$. aculeatum Parona et Perugia, 1889, ventral view; $\mathbf{C}$ - musculature of anchors and bars of $D$. aculeatum, dorsal view. D - anchoral musculature of D. aculeatum, ventral view. Colour codes: grey - sclerites; red - muscles. For Abbreviations see pages 2 and 3.

the same point as vad3 (arrow, Fig. 3D) and inserts on the lateral tip of the bar.

The group inserting on the body wall comprises two extrinsic muscles (daw and vaw). Daw (Figs. 2B-D, 4B) is a slender muscle that originates from the tip of the outer root of the dorsal anchor and passes forward through the peduncular region to insert on the body wall in the posterior body proper. The fibres of vaw (Figs. 2B-D, 3A,D, 5C, $6 \mathrm{~A})$ radiate from a narrow origin on the tip of the outer root of the ventral anchor and then run anteromedially in a par- allel fashion within the body wall to terminate just anterior to the peduncular region.

The group interconnecting the ipsilateral anchors of different pairs comprises two muscles (ivd1 and $i v d 2$ ). Ivd1 (Figs. 2D, 3A,D) is a slender muscle spanning between the outer root of the dorsal anchor and the inner root of the ventral anchor. $I v d 2$ (Figs. 2C,D, 3A-E, 4A) interconnects the inner roots of the dorsal and ventral anchors.

The muscle group inserting on the anchoral openings includes two muscles (vao, Figs. 2C,D, 3A,D, 6C; dao, 

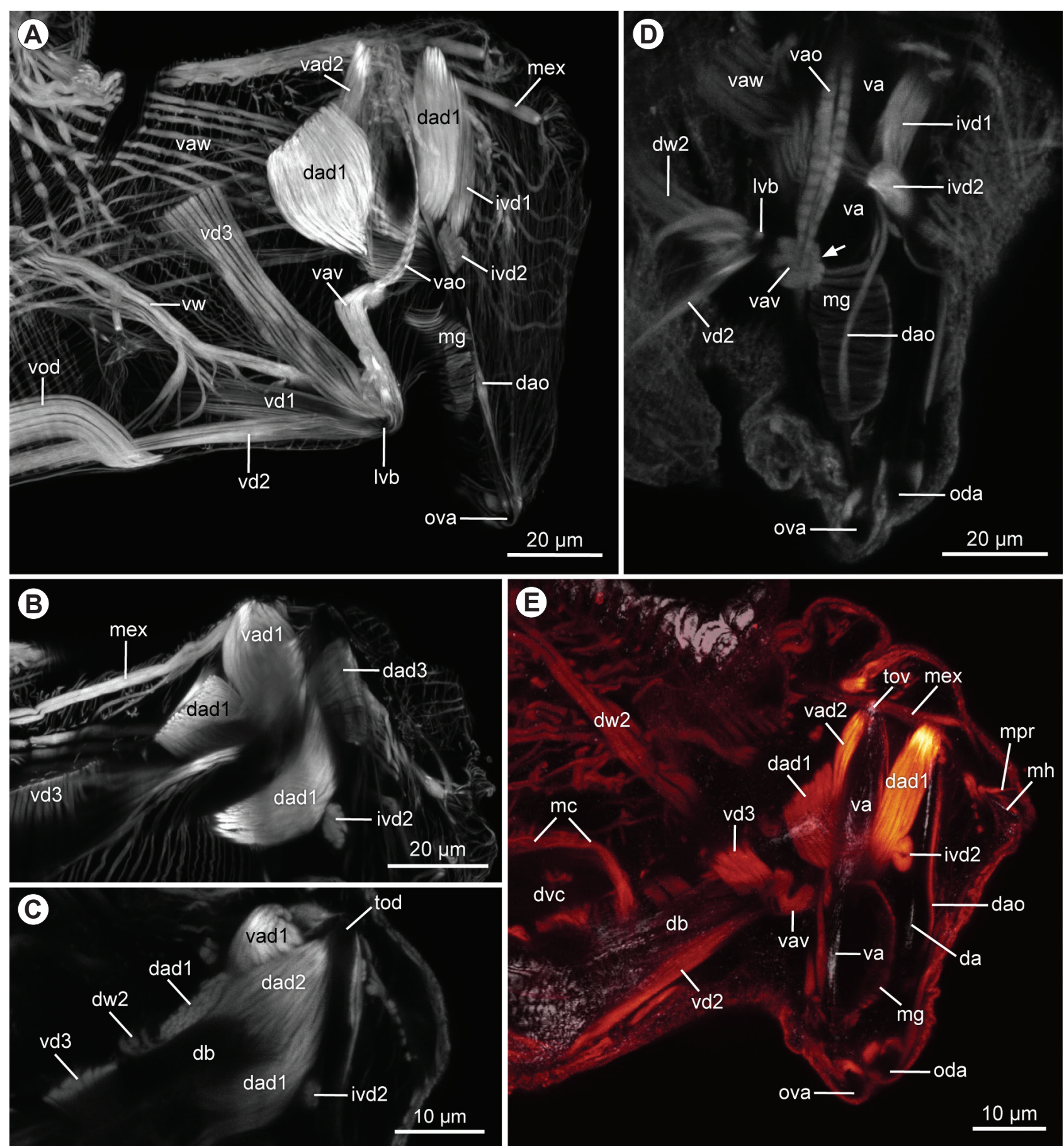

Fig. 3. Confocal images of the haptoral musculature of species of Diplectanum Diesing, 1858 from Sciaena umbra Linnaeus. A - musculature of anchors and connecting bars of $D$. similis Bychowsky, 1957; B - anchoral musculature of D. similis; $\mathbf{C}$ - musculature of anchors and the connecting bars of $D$. sciaenae van Beneden et Hesse, 1863; D - anchoral musculature of D. sciaenae. Note the striation of the vao-muscle. Arrow shows the position of a presumable insertion of the vav-muscle on the ventral anchor; $\mathbf{E}-$ sclerites and haptoral musculature of $D$. sciaenae. Colour codes: orange - phalloidin; grey - reflected light. For Abbreviations see pages 2 and 3.

Figs. 2C,D, 3A,D,E, 4A) that originate from the tip of the outer root of ventral ( $\mathrm{vao}$ ) or dorsal (dao) anchors and terminate on the body wall at the opening of the corresponding anchor. Muscle vao, unlike all other muscles in the haptor, is distinctly cross-striated (Fig. 3A,D), with a periodicity of 1.2-1.4.

The ventral anchors in all three species are associated with a large bladder-like structure that lies alongside the anchoral blade and is surrounded by a coat of closely set circular muscles ( $m g$, Figs. 3A,D,E, 4A,B, 5A,C). This bladder appears to open outside at the opening of the anchor and most likely represents the muscular reservoir of an anchoral gland.

Musculature of the bars. The muscles of the bars can be divided into four categories: the muscles originating from the anchors (described in the previous section), the muscles interconnecting the bars, the muscles inserting on the body wall and those attached to the anchoral openings. 

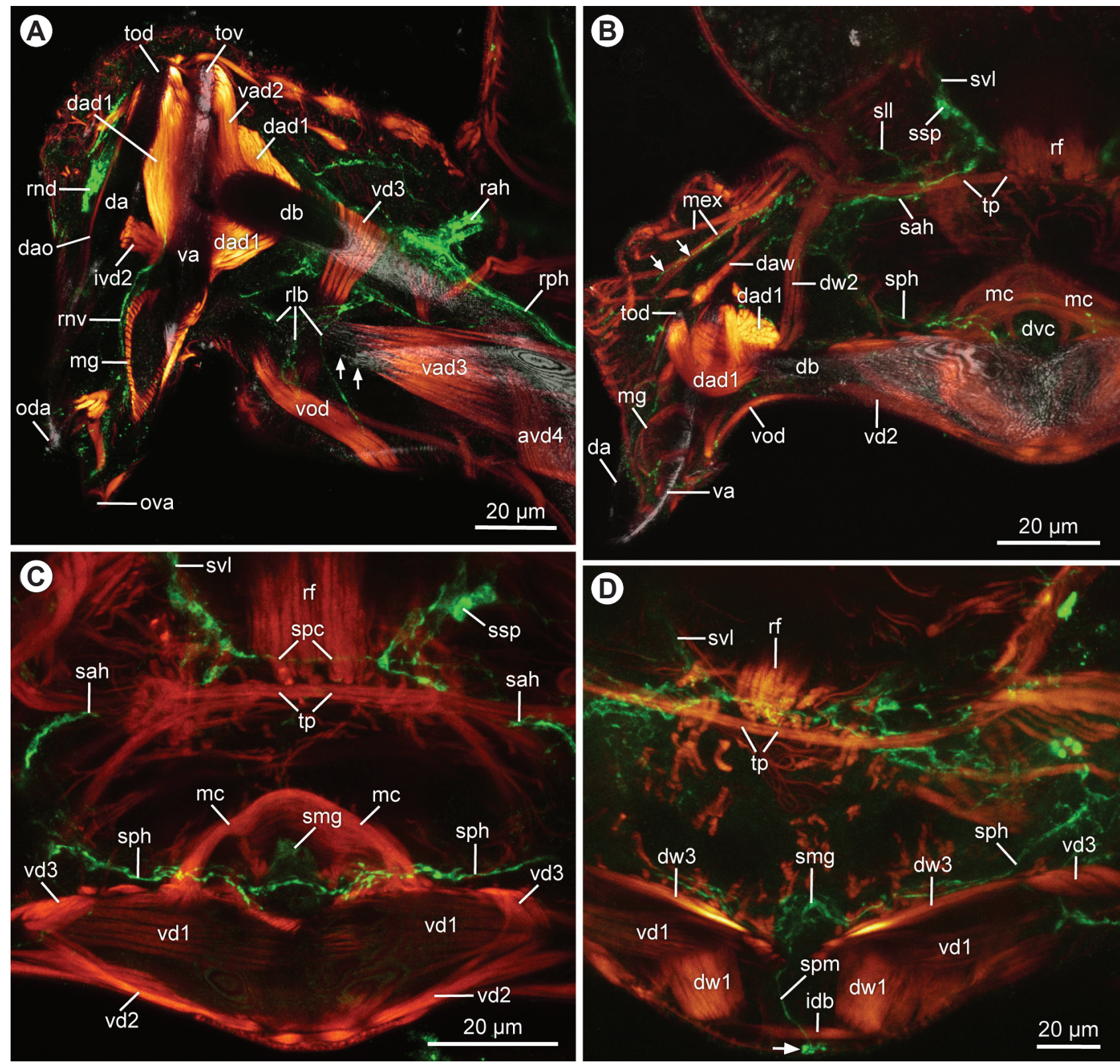

Fig. 4. Confocal images of the haptoral musculature, nervous system and sclerites of species of Diplectanum Diesing, 1858 from Sciaena umbra Linnaeus. A - musculature and RF-IR of anchors and bars of D. similis Bychowsky, 1957. Arrows indicate the tendinous projection of vad3. B - musculature and 5HT-IR of the haptor of D. sciaenae van Beneden et Hesse, 1863. Arrows indicate the neurites running towards the marginal hooks along their extrinsic muscles (mex). $\mathbf{C}-$ central portion of the haptor of $D$. sciaenae showing the haptoral musculature and 5HT-IR. D - central portion of the haptor of D. aculeatum Parona et Perugia, 1889 showing the musculature of the connecting bars and 5HT-IR. The arrow indicates varicosities on the posterior neurite (spm) projecting from the medial ganglion (smg). Colour codes: orange - phalloidin; green - 5HT-IR and RF-IR; grey - reflected light. For Abbreviations see pages 2 and 3.

All of these muscles, except the medial $i d b$-muscle, are bilaterally paired.

The musculature interconnecting the dorsal and ventral bars is the most prominent muscle group consisting of four muscles (vd1-4). $V d 1$ (Figs. 2C, 3A, 4C,D, 5A,B) begins as a loose band of fibres, with a wide attachment to the medial edge of the dorsal bar; the fibres of this muscle continue laterally over the dorsal surface of the ventral bar and converge on a narrow attachment point at the lateral tip of this bar. $V d 2$ (Figs. 2C, 3A,D,E, 4B,C, 5B, 6C) is a slender, compact bundle that spans from the lateral tip of the ventral bar to a triangular protrusion on the posteromedial corner of the dorsal bar, which is especially prominent in $D$. aculeatum (mpp, Fig. 1G,H).

$V d 3$ (Figs. 2B,C, 3A-C,E, 4A,C,D, 5A,D) connects the lateral tip of the ventral bar to the dorsal bar. In D. similis, the fibres of this muscle extend from the ventral bar more or less anteriorly towards the dorsal bar (Figs. 3A, 4A), bend sharply over the anterior margin of this bar and flare out posteriorly (Fig. 3B) to insert at the posterior edge of the bar just medial to the insertions of the anchoral muscles. In D. aculeatum and D. sciaenae, the morphology of this muscle is different: its fibres run from the ventral bar diagonally in an anteromedial direction (Figs. 2B, 3E, 

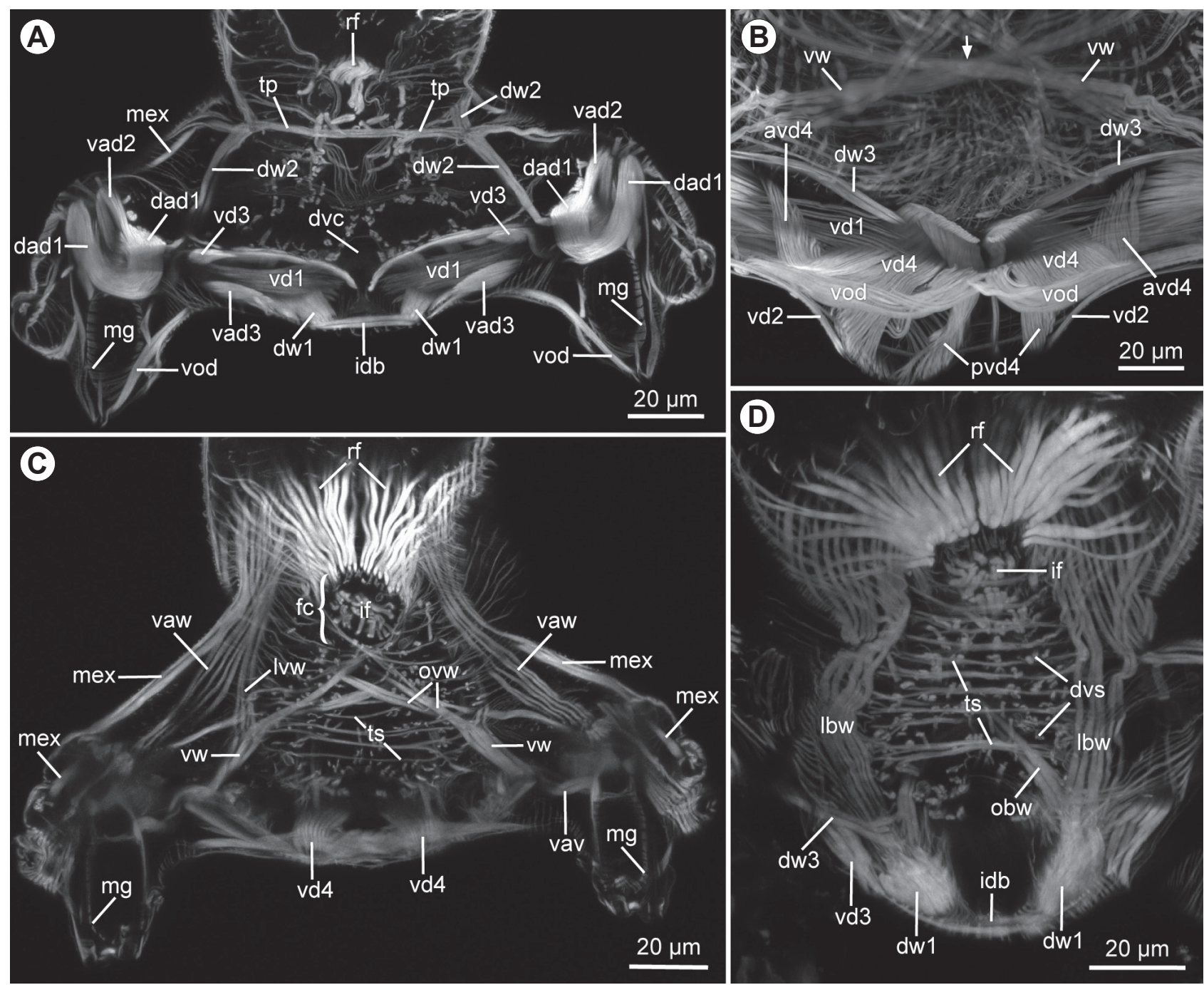

Fig. 5. Confocal images of the haptoral musculature of species of Diplectanum Diesing, 1858 from Sciaena umbra Linnaeus. A - haptor of D. aculeatum Parona et Perugia, 1889. Note that the dorsoventral cylinder (dvc) in this species lacks the thick coat of circular muscles; B - posterior portion of the haptor of $D$. similis Bychowsky, 1957 showing the musculature of the bars. Arrow indicates the point where contralateral vw-muscles cross each other at the haptor midline; $\mathbf{C}$ - haptor of D. aculeatum. Note numerous thin dorsoventral muscles seen as cross-sections in the interspaces between the ts-muscles; $\mathbf{D}$ - central portion of the haptor of $D$. sciaenae van Beneden et Hesse, 1863. For Abbreviations see pages 2 and 3.

4C,D, 5A), bend over the dorsal bar (Fig. 3C) and then continue posteromedially converging on a narrow point at the lateral face of the medial extension of the bar (Figs. 2C, 5D). $V d 4$ (Figs. 2B, 5B,C, 6C) is a wide, bracket-shaped muscle with both ends attached to the dorsal bar. Although $v d 4$ has no direct connection to the ventral bar, this muscle is grouped with the interconnecting muscles, because it makes a tight loop around the ventral bar, thereby holding both bars together. In $D$. similis, $v d 4$ begins as a loose bundle of fibres on the medial margin of the dorsal bar; the fibres converge anterolaterally, wrap around the anterior margins of both bars, continue posteriorly across the surface of the ventral bar and then split into two branches. One of these branches extends posteriorly to terminate on the posterior margin of the dorsal bar ( $p v d 4$, Fig. 5B) and the other ( $a v d 4$, Figs. 4A, 5B) bends again over the posterior edge of the ventral bar and attaches to the same oblique ridge on the dorsal bar as vad3. In D. aculeatum and $D$. sci- aenae, $v d 4$ (Figs. 2B, 5C, 6C) is not divided into branches and attaches fleshly to the anteromedial and posteromedial edges of the dorsal bar.

In $D$. aculeatum and $D$. sciaenae, the opposite dorsal bars are interconnected with a slender muscle (idb, Figs. 2B,C, 4D, 5A,D) that spans between the posteromedial corners of the bars anchoring to the same triangular projections as $v d 2$ (mpp, Fig. $1 \mathrm{G}, \mathrm{H})$. This muscle appears to be absent in $D$. similis.

The bars are connected to the body wall with four muscles ( $d w 1-3$ and $v w) . D w 1$ (Figs. 2B,C, 4D, 5A,D) is a short wedge-shaped muscle that originates from the posteromedial corner of the dorsal bar and runs anteriorly to insert on the body wall in front of the bars. Two bands of body-wall muscle fibres arise from the insertion of $d w 1$ : a longitudinal band (lbw, Figs. 2B,C, 5D, 6D) extending forward beyond the peduncular region to end in the posterior body proper and a diagonal band (obw, Figs. 2B,C, 5D, 

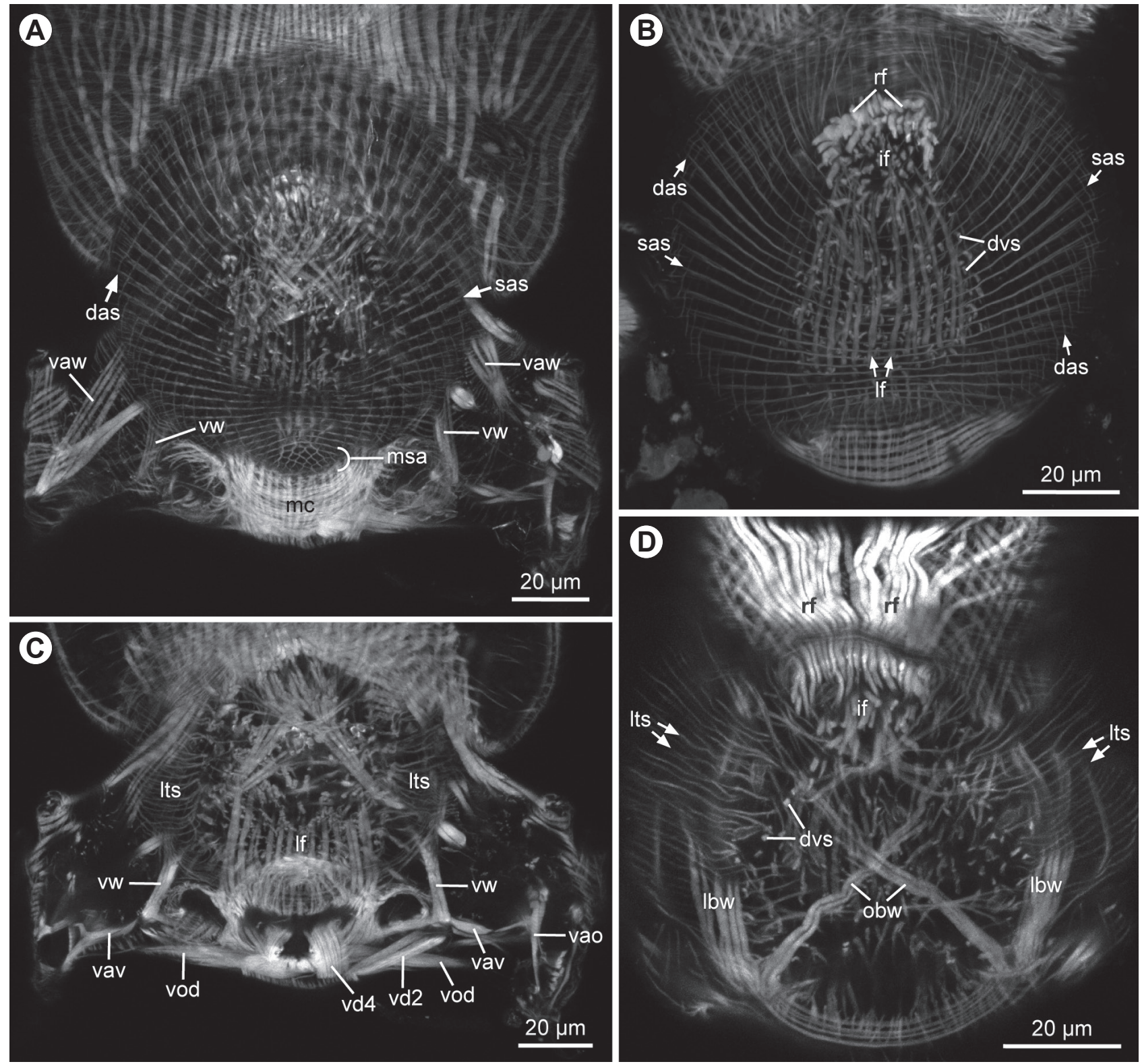

Fig. 6. Confocal images of the haptor showing the squamodisc musculature of species of Diplectanum Diesing, 1858 from Sciaena umbra Linnaeus. A, B - musculature of the ventral squamodisc of D. sciaenae van Beneden et Hesse, 1863; $\mathbf{C}$ - haptoral musculature of D. sciaenae; D - dorsal view of the haptor of D. aculeatum Parona et Perugia, 1889 showing tapering lateral endings (1ts) of the transverse muscles. For Abbreviations see pages 2 and 3.

6D) that crosses over to the contralateral side behind the squamodisc fulcrum. Dw2 (Figs. 2B-D, 3C-E, 4B, 5A) is present only in D. aculeatum and $D$. sciaenae. This muscle originates from the anterior edge of each dorsal bar just lateral to the insertions of the anchoral muscles and extends forward to end on the lateral body wall anterior to the peduncular region. Dw3 (Figs. 2B,C, 4D, 5B,D) arises anterolaterally from the dorsal bar and is overlaid at its origin by $d a d 1$. This slender muscle runs medially along the anterior margin of the dorsal bar and ends on the body at the insertion of $d w 1$.

The morphology of $v w$ differs in $D$. similis from that in the other two species. In D. aculeatum and D. sciaenae ( $v w$, Figs. 2B,C, 5C, 6A,C), this muscle takes its origin from the lateral tip of the ventral bar and runs anteromedially to split into two branches, one of which (ovw, Figs. 2B,C, 5C) continues diagonally, crosses its bilateral counterpart behind the squamodisc fulcrum and inserts on the body wall lateral to the fulcrum, and the other ( $l v w$, Figs. 2B,C, 5C) extends longitudinally, exits the haptor and ends on the body wall in the posterior body proper. In D. similis, $v w$ (Figs. $3 \mathrm{~A}, 5 \mathrm{~B}$ ) begins as an array of ramifying branches attaching to the ventral bar over much of its posterior margin; these branches converge into a single strand that runs diagonally beyond the haptor to terminate in the posterior body proper on the opposite side of the body wall.

The musculature attaching to the anchoral openings comprises one muscle (vod, Figs. 2B-D, 3A, 4A,B, 5A,B, 

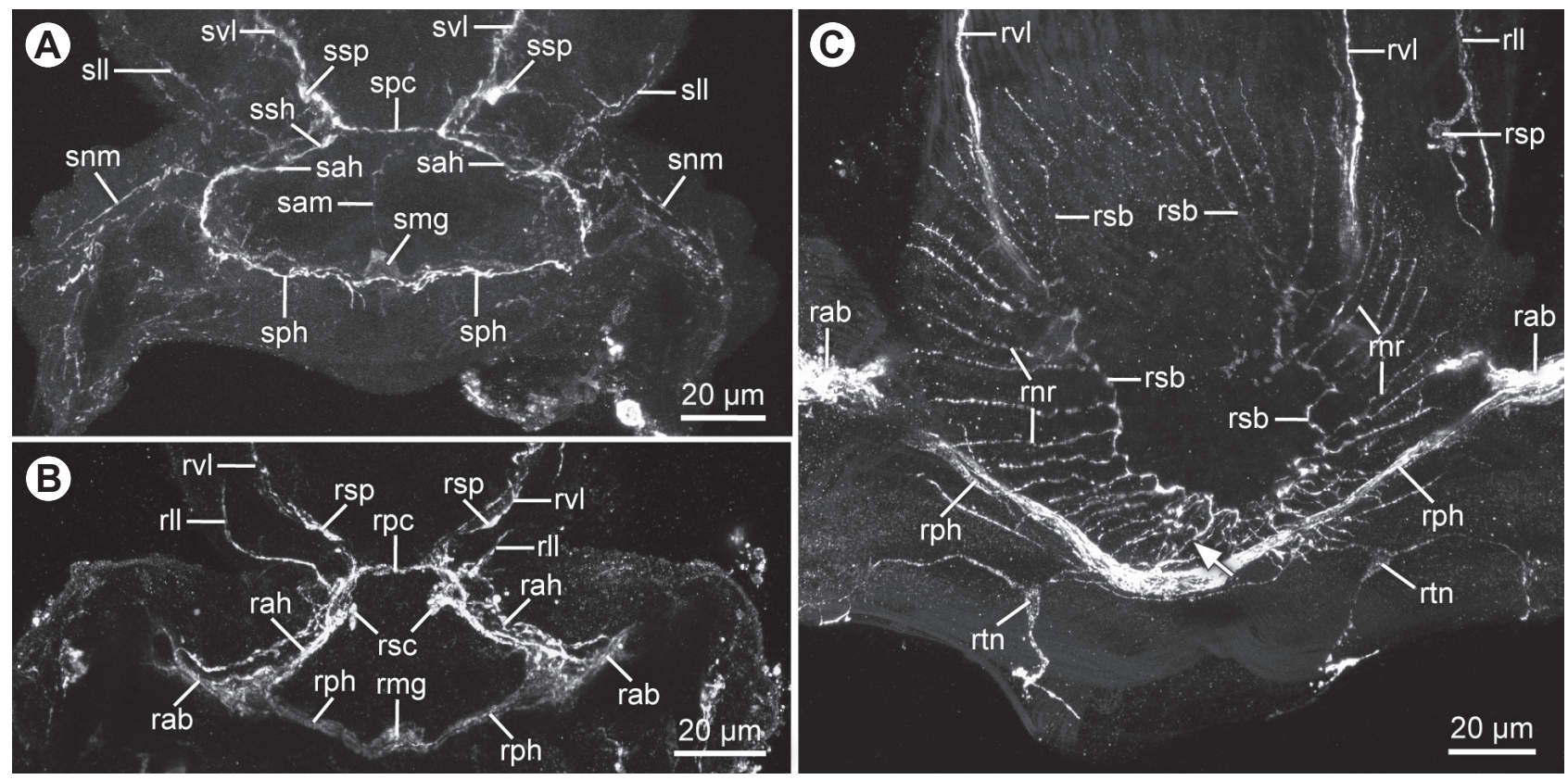

Fig. 7. Confocal images of the haptoral nervous system of species of Diplectanum Diesing, 1858 from Sciaena umbra Linnaeus. A - 5HT-IR nervous system of the posterior body proper and haptor of $D$. sciaenae van Beneden et Hesse, 1863; B - RF-IR nervous system of the posterior body proper and haptor of D. aculeatum Parona et Perugia, 1889; C - RF-IR of the haptor of D. similis Bychowsky, 1957 showing the arborisation of the neurite (arrow) supplying a squamodisc. For Abbreviations see pages 2 and 3.

6C) beginning from the medial knob of the ventral bar and extending laterally along the posterior margin of the haptor to end at the opening of the ventral anchor.

Musculature of marginal hooks. The individual muscles of the marginal hooks are difficult to identify because of their minute size, but it is clear that all marginal hooks have a few small intrinsic muscles and two protractor muscles ( $m p r$, Fig. 3E) extending diagonally from the proximal end of the hook to the body wall around the hook's opening. Each of the five pairs of hooks located at the lateral margins of the haptor is associated with an extrinsic muscle (mex, Figs. 3A,B,E, 4B, 5A,C) that anchors at the wall of the hook's papilla and extends anteriorly into the body proper to attach to the body wall just anterior to the peduncular region. The two middle pairs of marginal hooks lack extrinsic muscles.

Musculature of squamodiscs. The most pronounced musculature of each squamodisc consists of about 20-25 myofibres (rf, Figs. 2A, 4B-D, 5A,C,D, 6B,D) originating in a semicircle along the anterior periphery of the fulcrum; these muscle fibres radiate anteriorly to end at the body wall in the posterior body proper. A thick bundle of muscle fibres (if, Figs. 2A, 5C,D, 6B,D) connects the centres of the fulcra of the opposite squamodiscs. Another bundle of fibres (lf, Figs. 2A, 6B,C) arises from the posterior rim of the fulcrum and extends longitudinally in a slightly radiating fashion towards the posterior end of the squamodisc.

Numerous thin dorsoventral myofibres ( $d v s$, Figs. 2A, $5 \mathrm{D}, 6 \mathrm{~B}, \mathrm{D})$ span between the posterior regions of the opposite squamodiscs attaching to the squamodiscs in the interspaces between the $l f$-muscles. Each arch of the squamodisc ossicles posterior to the fulcrum is associated with a thin bundle of myofibres (sas, Figs. 2A, 6A,B), which follow the contours of the arches and are likely to pass through the internal rodlet-shaped portions of ossicles. The sas-muscles form U-shaped arches behind the fulcrum covering most of the squamodisc area in parallel rows and gradually changing their shapes from distinctly arcuate anteriorly to only slightly curved posteriorly.

Another layer of thin arcuate muscle bundles (das, Figs. 2A, 6A,B) lies just underneath the sas-muscles. Unlike the sas-muscles, these bundles arc in front of the fulcrum forming forward-facing arches across the squamodisc. The das-muscles are followed internally by numerous transverse muscles ( $t s$, Figs. 2A, 5C,D), crossing the interior of the haptor, with one larger transverse muscle ( $t p$, Figs. 4B-D, 5A) spanning the haptor at the peduncular region. The lateral portions of the $t s$-muscles (lts, Fig. 6C,D) are gradually tapering to their ends and anchor at the lateral margins of the squamodiscs.

The squamodiscs of all three species are associated with a dorsoventral cylindrical strand of tissue (dorsoventral cylinder, $d v c$, Figs. 3E, 4B, 5A) that stretches medially between the posterior portions of the opposite squamodiscs abutting on the anterior medial notch of the ventral bar. In $D$. sciaenae, this structure is surrounded by a thick coat of circular muscles ( $m c$, Figs. 3E, 4B,C, 6A) and three or four posterior rows of ossicles where the cylinder attaches to the squamodiscs have a modified arrangement of the sas-muscles (msa, Figs. 2A, 6A): the fibres of these muscles surround each individual ossicle forming a meshwork-like pattern, rather than the transverse arches like the more anterior muscles.

Serotonin-immunoreactive nervous system of the haptor. 5HT-IR neurites in the posterior body proper are arranged in a pair of ventral bundles ( $s v l$, Figs. 4B-D, 7A) 
composed of 3-4 neurites and a pair of lateral bundles (sll, Figs. 4B, 7A) consisting of two neurites. At the peduncular region, the ventral bundles run diagonally towards each other (Figs. 4C, 7A) and are connected by a ventral commissure (spc, Figs. 4C, 7A). At the origin of this commissure the ventral bundle turns laterally and merges with the lateral bundle (Figs. 4B, 7A). Two or three cell bodies (ssp, Figs. 4B,C, 7A) are located within the ventral bundles just anterior to the commissure and there are also one or two neuronal somata (ssh, Fig. 7A) at the fusion of the bundles, but these cells do not appear to form a well-defined ganglion.

The fused bundles proceed into the haptor, where they unite in a closed dorsal loop (Figs. 4C, 7A). In this loop, the contralateral bundles first extend laterally ( $s a h$, Figs. 4B,C, 7A), then curve sharply medially and run towards each other along the anterior margins of the dorsal bars (sph, Figs. 4B-D, 7A) meeting at a medially located ganglion (smg, Figs. 4C,D, 7A) composed of two or three somata. The ganglion is situated topographically inside the dorsoventral cylinder and, in D. sciaenae, is surrounded by the circular muscles of the cylinder (Fig. 4C).

One of the cells of the medial ganglion projects a single posterior neurite (spm, Fig. 4D) along the haptor midline towards the posterior end of the haptor where it forms a cluster of varicosities between the dorsal bars (arrow, Fig. 4D). Another neurite projects from the ganglion anteriorly (sam, Fig. 7A).

Laterally, the haptoral loop gives rise to the neurite bundles that run towards the anchors. Near the anchors, these bundles divide into two, with one branch running along the ventral and the other along the dorsal anchor.

The neurite bundles (arrows, Fig. 4B; snm, Fig. 7A) supplying the marginal hooks arise from the lateral aspects of the haptoral loop anterior to the anchoral branches and extend towards the marginal hooks along their extrinsic muscles.

RF-immunoreactive nervous system of the haptor. RF-IR neurites form two pairs of longitudinal neurite bundles, the ventral bundles consisting of three neurites ( $r v l$, Fig. 7B,C) and the lateral bundles of one or two neurites (rll, Fig. 7B,C). The general arrangement of the bundles in the posterior body proper and the haptor is similar to that of the 5HT-IR cells: at the peduncular region, the ventral bundles are interconnected by a commissure ( $r p c$, Fig. 7B) and, more posteriorly, fuse with the lateral bundles (Fig. 7B); the fused bundles form a dorsal loop (Figs. 4A, 7B,C) in the haptor with a medial ganglion ( $r m g$, Fig. 7B) at its posterior pole. There is one or possibly two cell bodies ( $r s p$, Fig. 7B,C) just anterior to the connecting commissure and at the fusion of the ventral and lateral bundles ( $r s c$, Fig. 7B) and one or two somata within the medial ganglion (rmg, Fig. 7B). The anchoral bundles have the same morphology as those revealed by 5HT-IR fluorescence: they arise laterally from the haptoral loop ( $r a b$, Fig. 7B,C) and divide into two branches running along each anchor (rnd, Fig. 4A).

The medial portion of the posterior arc of the loop gives rise to two neurites (arrow, Fig. 7C) that run anteriorly, one close to the ventral and the other to the dorsal side of the haptor, each supplying the corresponding squamodisc. In the posterior portion of the squamodiscs, these neurites bifurcate into two meandering side branches ( $r s b$, Fig. 7C) that course in a wide arc towards the anterior end of the squamodisc staying about halfway between the midline and the lateral edges of the squamodisc. Along their length, these side branches give off lateral offshoots ( $r n r$, Fig. 7C) that follow laterally the curvature of each row of ossicles. Judging from the arrangement of these neurites, the whole set of branches supplying each squamodisc might constitute an extensive neuritic arborisation of a single neuron.

A pair of tripolar nerve cells (rtn, Fig. 7C) lies submedially on the dorsal side near the posterior margin of the haptor. One of the neurites of these cells projects posteriorly over the medial portion of the dorsal bar, another proceeds laterally and the third neurite extends anteriorly to join the haptoral loop. Laterally, one or possibly two neurites project posteriorly from the haptoral loop and branch out over the lateral end of the ventral bar (rlb, Fig. 4A).

\section{DISCUSSION}

The results of the present study add new details to the earlier descriptions of anchoral musculature in species of Diplectanum (see Paling 1966, Oliver 1969, 1987). Paling (1966) described six anchoral muscles in D. aequans and Oliver $(1969,1987)$ added a description of 12 other muscles in his account on D. aculeatum and D. sciaenae. We found 23 major muscles associated with anchors and connecting bars in D. aculeatum and D. sciaenae and 21 muscles in D. similis, of which six in all three species ( $d a d 2$, $d a d 3, i v d 1$, vaw, vod and $d w 2$ ) clearly correspond to those listed by Oliver (his muscles VII, VIII, IX, VI, XI and XIII, respectively). The remaining muscles do not appear in Paling or Oliver's accounts or cannot be unambiguously matched to those described by these authors. The complex looping configuration of dadl makes it difficult to describe this muscle correctly from histological sections and it is possible that it was described by Oliver as two independent muscles (I and X). Paling (1966) recognised the looping of the dad1-muscle (his muscle I), but in his description it wraps around the dorsal anchor rather than the dorsal bar. Paling (1966) and Oliver $(1969,1987)$ have also reported two short muscles between the dorsal bar and the dorsal anchor (III and IV in their notation), but these muscles were not seen in any of the three species examined by confocal microscopy and it cannot be ruled out that these are in fact ligaments connecting the anchor to the bar.

The new information on anchoral musculature presented in the present study allows for more accurate predictions about the mechanics of attachment in Diplectanum and the specific roles of individual anchoral muscles. It is clear that the anchoral musculature of the Diplectanidae has been modified from the more plesiomorphic condition found in other dactylogyroideans studied to date, such as the members of the Dactylogyridae Yamaguti, 1963 or Ancyrocephalidae Bychowsky et Nagibina, 1968 (Kearn 1971, Gerasev 1977, 1981, 1989, Petrov et al. 2015, 2016). In these dactylogyroideans, a significant role in the gaffing 
action is played by the transverse muscles connecting the opposite anchors and by large extrinsic muscles that often extend all the way to the anterior end of the body. In most cases, the extrinsic or transverse muscles appear to function as catch muscles holding the anchors in the attached position.

In the Diplectanidae, the powerful musculature of the adhesive discs stands in the path of the extrinsic muscles, rendering them inefficient as catch muscles. The muscles of Diplectanum that may be homologous to the extrinsic muscles (vaw and daw) do not extend much further than the peduncular region apparently playing only a secondary part in the movement of the anchors and can be viewed as vestigial remnants of the more powerful extrinsic musculature of other Dactylogyroidea. The transverse muscles would also be inefficient as catch muscles in the Diplectanidae, because of a wide distance between the contralateral anchors; these muscles are completely absent in Diplectanum. Apparently, the Diplectanidae have adopted a different type of catch mechanism where extrinsic or transverse muscles were replaced by those attached directly to the connecting bars.

The large size and the shape of the dadl-muscle make it the best candidate for the role of a catch muscle in $\mathrm{Di}$ plectanum. Judging from the configuration of this muscle, it probably pulls the tip of the outer root of the dorsal anchor medially towards the dorsal bar and pivots the anchor around its ligamentous connection to the bar so that the anchoral blade is rotated outwards to gaff the host tissues. As the dad1-muscle overlays the vad2-muscle, it may clamp this muscle against the bar, which probably also results in the outward rotation of the ventral anchor. In this way, the dad1-muscle may hold both anchors attached in the host tissue and its looping position around the dorsal bar may serve to increase the power with which the anchors are held. The function of another muscle of the dorsal anchor, dad2, is likely to reinforce the action of dad1 acting as a supplementary catch muscle for this anchor and vadl may play the same role for the ventral anchor. Removal of the ventral anchor from the host tissue is probably accomplished by three muscles originating from the ventral bar (vod, vav and vad3). There are no muscles that can perform the same function for the dorsal bar and it is possible that this anchor is removed from the tissue by elastic recoil of the ligaments once the catch muscles are relaxed. The removal of this anchor may also be assisted by contraction of the $v d 3$-muscle resulting in the pivoting of the dorsal bar with respect to the ventral bar. When this muscle contracts, it draws the tip of the dorsal bar towards the ventral bar pulling the anchor posteriorly and relaxing its hold on the host tissues. The remaining anchoral muscles are probably less important in attachment and provide fine motor control for the anchors.

The function of the vao muscle is of special interest, because it differs from all other haptoral muscles, including its dorsal counterpart ( $\mathrm{dao}$ ), in being distinctly cross-striated. Striated muscles have been described in the haptors of several monopisthocotyleans (e.g. Kearn et al. 1990, 2003) and their presence in monogeneans and other soft-bodied invertebrates appears to be related to the requirement for fast contraction over a limited action length (Kearn et al. 2003, Schmidt-Rhaesa 2007). It is unlikely that the vao muscles play any significant role in anchoral movement and their function may be associated with the anchoral glands, whose muscular reservoirs $(m g)$ open near the insertion of these muscles at the openings of the ventral anchors. If the anchoral glands have an adhesive function, it can be surmised that the fast twitch of the vao muscles may detach the anchoral glands from the substrate when the worm changes its position on the gills.

The attachment of the squamodiscs has been described by several authors (Paling 1966, Oliver 1969, 1987, Dezfuli et al. 2007, Sánchez-García et al. 2011) and these observations provide a framework for our inferences on the functional roles of the squamodisc muscles. In detached worms, the squamodiscs make repeated grasping movements in which their anterior regions are cupped and bent posteriorly and then rapidly retracted to lie closely apposed to the body wall (Paling 1966). When attached, the worms keep their squamodiscs in a protracted position. The worms lodge themselves between two adjacent secondary lamellae by the antagonising actions of the squamodiscs and the anchors: the anterior regions of the squamodiscs push the worm out, while the gaffing action of the anchors pulls it deeper into the lamellae balancing out the force exerted by the squamodiscs (Paling 1966). Oliver $(1969,1987)$ argued that the contraction of the squamodisc muscles lifts the central region of the squamodisc off the surface creating a pocket of low pressure and so the squamodiscs may also function as suction cups.

The arrangement of the squamodisc muscles described in the present study is in good agreement with Paling's (1966) observations. The functioning of the squamodiscs as suction cups seems less likely, because the complex shapes of ossicles make suction inefficient, although a certain amount of suction force may still be present. The musculature attaching to the fulcrum may either cup or pivot the squamodisc depending on which muscles are engaged in the action. The forward pivoting and protraction of the squamodisc is most likely accomplished primarily by the $r f$-muscles. The if-muscles can play a subsidiary role in squamodisc protraction: their contraction brings the fulcra of the squamodiscs towards each other allowing the $r f$-muscles to pull the fulcrum forward without shrivelling or buckling the anterior portion of the squamodisc. It is also possible that when the animals are lodged in the host tissues, the if-, $r f$ - and $l f$-muscles are all maintained simultaneously in the contracted state, with the $r f$ - and $l f$-muscles balancing each other out by pulling the fulcrum in the opposite directions.

The arrangement of the superficial sas- and das-muscles and their apparent association with ossicles raises a strong possibility that these muscles are responsible for the movement of individual ossicles. If the adjacent ossicles in the same row are spaced loosely when the sas-muscles are relaxed and the squamodisc surface is stretched by the fulcrum muscles, the contraction of the sas-muscles would close the gaps clamping the host tissue between 
the ossicles and reinforcing the purchase on the surface of the lamella. In the same way, the das-muscles may close the spaces between the ossicles in the adjacent rows. The $t s$-muscles probably anchor laterally on the peripheral tegumental fold of the squamodisc and may function similarly to the sas-muscles tightening the spacing between the ossicles when the squamodisc is closely applied to the surface of the gill.

The three species of Diplectanum show clear differences in the arrangement of the haptoral muscles, which are consistent across all specimens. Most of the haptoral muscles of $D$. aculeatum and $D$. sciaenae are identical and these two species differ only in the presence of a muscular sheath around the cylindrical tissue strand between the squamodiscs in D. sciaenae and in the different patterns of the posterior sas-muscles. The function of the muscular sheath in D. sciaenae is unclear but its association with the squamodiscs suggests that contraction of its circular muscles may push apart the posterior regions of the opposite squamodiscs. In this way, the posterior squamodisc regions may act in concert with the anchors pulling the worm deeper between the gill lamellae in opposition to the action of the anterior regions of the squamodiscs. The meshwork pattern of the posterior sas-muscles in D. sciaenae may also be associated with this presumable function of the muscular sheath. Another possible function of the muscular sheath is protection of the medial ganglion that might be under a significant mechanical stress when the muscles of the squamodisc fulcrum are in the contracted state.

Diplectanum similis has anchoral muscles identical to those of the other two species, but lacks two of the muscles (idb and $d w 2$ ) present in D. aculeatum and D. sciaenae and also differs significantly in the shapes, trajectories and attachment points of two muscles interconnecting the bars ( $v d 3$ and $v d 4$ ) and one muscle connecting the dorsal bar to the body wall $(d w 3)$. These differences are likely to be explained by a somewhat different angle between the ventral and dorsal bars in D. similis, requiring different vectors of forces acting on the bars and, as a result, a different configuration of musculature compared to the other two species.
The architecture of the haptoral nervous system in $\mathrm{Di}$ plectanum appears to be consistent with what has been observed in other representatives of the Dactylogyroidea (Buchman and Prento 1989, Timofeeva and Kotikova 1993, 1995, Petrov et al. 2016). The arrangement of the nervous elements in the haptor is rather uniform throughout this group. In all studied dactylogyroideans, the lateral and ventral nerve cords merge in front of the haptor; there is a pair of ganglia near or at the confluence of the cords and the opposite cords are connected by a commissure. The fused cords continue into the haptor where they form a loop with two or several ganglia, which supplies nerves to the anchors and marginal hooks. The only major addition to this common plan in Diplectanum is a distinct RFamide-IR pattern of neurite arborisation associated with squamodiscs, the attachment structures unique for Diplectanidae. Lateral offshoots in this arborisation pattern parallel the rows of ossicles and probably are involved in the motor control of the superficial squamodisc muscles.

In general, the haptoral musculature of Diplectanum holds substantially more promise as an additional morphological criterion in genus-level taxonomy than does the nervous system. There are clear differences in the arrangement of the haptoral muscles between all three species, which are highly consistent across the specimens studied. The RFamide- and 5HT-immunoreactive nervous systems show very little variation between species and there is also slight inconsistency between the specimens due to differences in staining intensity, which makes interspecific comparison difficult. The nervous system of Diplectanum appears to be evolutionarily more conserved than the musculature conforming to the general pattern of the nervous system in the Dactylogyroidea and as such may be useful as a taxonomic criterion at the level of higher-rank taxa rather than at the generic level.

Acknowledgements. This work was supported by budget funding (project AAAA-A17-117030110029-3) of the Russian Academy of Sciences and by the Russian Foundation for Basic Research (grants 14-04-90411, 15-29-02684 and 15-29-02650).

\section{REFERENCES}

Buchmann K., Prento P. 1989: Cholinergic and aminergic elements in the nervous system of Pseudodactylogyrus bini (Monogenea). Dis. Aquat. Organ. 6: 89-92.

Dezfuli B.S., Giari L., Simoni E., Menegatti R., Shinn A.P., MAnERA M. 2007: Gill histopathology of cultured European sea bass, Dicentrarchus labrax (L.), infected with Diplectanum aequans (Wagener 1857) Diesing 1958 (Diplectanidae: Monogenea). Parasitol. Res. 100: 707-713.

Domingues M.V., Boeger W.A. 2008: Phylogeny and revision of Diplectanidae Monticelli, 1903 (Platyhelminthes: Monogenoidea). Zootaxa 1698: 1-40.

Galli P., Pugachev O.N., Kritsky D. (Eds.) 2009: Guide to Monogenoidea of Freshwater Fish of Palaeartic and Amur Regions. Ledizione-Ledi Publishing, Milan, 564 pp.

Gerasev P.I. 1977: [The mechanism of attachment to the host's gills in Dactylogyrus extensus and D. achmerowi (Monogenoidea)]. Parazitologiya 11: 513-519. (In Russian.)
Gerasev P.I. 1981: [Muscle systems of haptors and the attachment mechanism of some species of Dactylogyrus (Monogenea, Dactylogyridae)]. Parazitol. Sb. 30: 190-205. (In Russian.)

Gerasev P.I. 1989: [Major muscles of anchors in Amuro-Chinese species of Dactylogyrus (Monogenoidea)]. In: B.I. Lebedev (Ed.), Parazitologicheskiye Issledovaniya. DVO AN SSSR, Vladivostok, pp. 39-45 (In Russian.)

Gibson D.I., Bray R.A. 2010: Diplectanum Diesing, 1858. In: S. Tyler, T. Artois, S. Schilling, M. Hooge and L.F. Bush (Eds.), World List of Platyhelminthes. World Wide Web electronic publication, http://www.marinespecies.org/aphia.php? $\mathrm{p}=$ taxdetails\&id=119291.

Justine J.-L., Henry É. 2010: Monogeneans from Epinephelus chlorostigma (Val.) (Perciformes: Serranidae) off New Caledonia, with the description of three new species of diplectanids. Syst. Parasitol. 77: 81-105.

KEARN G.C. 1971: The attachment of the ancyrocephalid monogenean Haliotrema balisticus to the gills of the trigger fish, Balistes capriscus (= carolinensis). Parasitology 63: 157-162. 
Kearn G.C., Beverley-Burton M. 1990. Mycteronastes undulatae gen. nov., sp. nov. (Monogenea: Monocotylidae) from the nasal cavities of Raja undulata in the Eastern Atlantic. J. Mar. Biol. Ass. UK 70: 747-753.

Kearn G.C., Whittington I., Evans-Gowing R. 2003. Striated (stratified) muscle and the operation of the ventral hamuli of the ancryocephaline monogenean Chauhanellus australis, from the gills of the blue catfish Arius graeffei. In: C. Combes and J. Jourdane (Eds.), Taxonomie, Écologie et Évolution des Métazoaires Parasites. Tome I. Presses Universitaires de Perpignan, Perpignan, pp. 381-396.

Oliver G. 1968: Recherches sur les Diplectanidae (Monogenea) parasites de Téléostéens du Golfe du Lion. I. Diplectaninae Monticelli 1903. Vie Milieu 19: 95-138.

Oliver G. 1969: Recherches sur les Diplectanidae (Monogenea) parasites de Téléostéens du Golfe du Lyon. III. Biology. Vie Milieu 20: 397-419.

Oliver G. 1980: Les Diplectanidae Bychowsky, 1957 (Monogenea, Monopisthocotylea) parasites des Sciaenidae (Pisces, Perciformes) du Golfe de Gascogne. Bull. Mus. Natl. Hist. Nat. 23 669-689.

Oliver G. 1987: Les Diplectanidae Bychowsky, 1957 (Monogenea: Monopisthocotylea, Dactylogyridea). Systematique. Biologie. Ontogénie. Ecologie. Essai de phylogenèse. Academie de Montpellier, Université des Sciences et Techniques du Languedoc, Montpellier, $443 \mathrm{pp}$.

Oliver G., Paperna I. 1984: Diplectanidae Bychowsky, 1957 (Monogenea, Monopisthocotylea) parasites de Perciformes de
Méditerranée orientale, de la Mer Rouge et de l'Océan Indien. B. Mus. Natl. Hist. Nat. 6: 49-65.

PALING J.E. 1966: The attachment of the monogenean Diplectanum aequans (Wagener) Diesing to the gills of Morone labrax L. Parasitology 56: 493-593.

Petrov A.A., Popyuk M.P., Dmitrieva E.V., Gerasev P.I. 2015: Architecture of haptoral musculature in three species of Ligophorus (Monogenea, Ancyrocephalidae). Trudy Zool. Inst. RAN 319: 244-256.

Petrov A.A., Popyuk M.P., Gerasev P.I., Dmitrieva E.V. 2016: Haptoral neuromusculature in two species of Dactylogyrus Diesing, 1850 (Monogenea: Dactylogyridae). Syst. Parasitol. 93: 337-354.

Sánchez-García N., Padrós F., Raga J.A., Montero F.E. 2011: Comparative study of the three attachment mechanisms of diplectanid monogeneans. Aquaculture 318: 290-299.

Schmidt-Rhaesa A. 2007. The Evolution of Organ Systems. Oxford University Press: Oxford, New York, 385 pp.

SHAw M.K. 1981: The ultrastructure of the pseudohaptoral squamodiscus of Diplectanum aequans (Monogenea). Parasitology 82: $231-240$.

Timofeeva T.A., Kotikova E.A. 1993: [Nervous system of the monogeneans of the orders Tetraonchoidea and Dactylogyroidea (Monogenea)]. Parazitologiya 27: 118-126. (In Russian.)

Timofeeva T.A., Kotikova E.A. 1995: [Topographical peculiarities of monogenean nervous systems]. Parazitologiya 29: 127 139. (In Russian.)

Cite this article as: Petrov A.A., Dmitrieva E.V., Popyuk M.P., Gerasev P.I., Petrov S.A. 2017: Musculoskeletal and nervous systems of the attachment organ in three species of Diplectanum (Monogenea: Dactylogyroidea). Folia Parasitol. 64: 022. 